TRANSACTIONS OF THE

AMERICAN MATHEMATICAL SOCIETY

Volume 357, Number 11, Pages 4633-4646

S 0002-9947(04)03614-1

Article electronically published on December 28, 2004

\title{
IRREGULAR HYPERGEOMETRIC SYSTEMS ASSOCIATED WITH A SINGULAR MONOMIAL CURVE
}

\author{
MARÍA ISABEL HARTILLO-HERMOSO
}

\begin{abstract}
In this paper we study irregular hypergeometric systems defined by one row. Specifically, we calculate slopes of such systems. In the case of reduced semigroups, we generalize the case studied by Castro and Takayama. In all the cases we find that there always exists a slope with respect to a hyperplane of this system. Only in the case of an irregular system defined by a $1 \times 2$ integer matrix we might need a change of coordinates to study slopes at infinity. In the other cases slopes are always at the origin, defined with respect to a hyperplane. We also compute all the $L$-characteristic varieties of the system, so we have a section of the Gröbner fan of the module defined by the hypergeometric system.
\end{abstract}

\section{INTRODUCTION}

Algebraic analysis or $\mathcal{D}$-module theory studies systems of linear partial differential equations from the point of view of algebra and it generalizes the classical theory of ordinary differential equations with holomorphic coefficients in one complex variable $x$.

A basic concept in the theory of ordinary linear differential equations with holomorphic coefficients is that of regular singular point. Fuch's theorem 8 gives a combinatorial method to determine when a singular point is regular or irregular. Given an ordinary linear differential equation

$$
a_{m}(x) \partial^{m}+a_{m-1}(x) \partial^{m-1}+\cdots+a_{0}(x), \text { with } a_{m}(x) \neq 0,
$$

we associate to it a combinatorial object, the Newton polygon, defined as

$$
\text { convex hull }\left(\bigcup_{j=0}^{m}\left(j, j-\operatorname{val}\left(a_{j}(x)\right)\right)+(-\mathbb{N})^{2}\right)
$$

where $\operatorname{val}\left(a_{j}(x)\right)$ is the order of the holomorphic function $a_{j}(x)$ at $x=0$.

Fuch's theorem assures that the ordinary differential equation has a regular singular point at the origin if and only if its Newton polygon is a quadrant. The notion of slope arises from this theorem: if the Newton polygon has a slope, then the origin is an irregular singular point. We say that the equation is regular if it has no irregular singular points.

Received by the editors July 15, 2003 and, in revised form, January 21, 2004.

2000 Mathematics Subject Classification. Primary 32C38; Secondary 13P10, 13N10, 33C80, $34 \mathrm{M} 35$.

Key words and phrases. $\mathcal{D}$-module, slopes, hypergeometric systems, Gröbner basis.

This work was partially supported by FQM-813, FQM-333, DGESIC BFM2001-3164 and HF2000-0044. 
To generalize the theory of linear partial differential equations we introduce the Weyl algebra:

$$
\mathcal{A}_{n}=\mathbf{C}\left\langle x_{1}, \ldots, x_{n}, \partial_{1}, \ldots, \partial_{n}\right\rangle,
$$

that is, the ring of differential operators with polynomial coefficients in $n$ variables. This ring is not commutative and the above elements verify the following relations: $\left[x_{i}, x_{j}\right]=0,\left[\partial_{i}, \partial_{j}\right]=0$, and $\left[\partial_{i}, x_{j}\right]=\delta_{i, j}$, where $\delta_{i, j}$ is the Kronecker delta.

We also need to introduce the ring $\mathcal{D}_{n}$ :

$$
\mathcal{D}_{n}=\mathbf{C}\left\{x_{1}, \ldots, x_{n}\right\}\left\langle\partial_{1}, \ldots, \partial_{n}\right\rangle
$$

with the same relations between the generators.

The first differential equation we considered can be seen as an operator in this ring. Every system of linear partial differential equations can be represented as a left ideal in $\mathcal{D}_{n}$, or in $\mathcal{A}_{n}$ if the coefficients are polynomial. This can be done because a solution of such a system is also a solution for every element which comes from a combination (as left ideal) of those operators.

In general we denote by $\langle M\rangle$ the left ideal generated by the elements of $M$. To simplify notation we usually note $\mathcal{A}, \mathcal{D}$ and $x$ and $\partial$ instead of $\left(x_{1}, \ldots, x_{n}\right)$ and $\left(\partial_{1}, \ldots, \partial_{n}\right)$, if there is no confusion.

The generalization of irregularity in several variables is not obvious. The equivalent notion is that of irregular sheaf, a concept due to Mebkhout (see 13]). Given a system, we consider the left ideal associated and the module defined by this ideal; if the irregular sheaf with respect to every hypersurface associated to this module is zero, we say that the system is regular.

There also exists a generalization of the concept of slope; it is due to Laurent [9] and it describes an invariant associated to a $\mathcal{D}$-module with respect to a hypersurface in one of its points.

As in the case of one variable, those concepts are related, for holonomic modules. The slopes of a module describe the jumps in the Gevrey filtration of that sheaf [10]. We have that a holonomic $\mathcal{D}$-module in several variables is regular if and only if it has no slopes for all the hypersurfaces ([10]).

The slopes are used very often to determine if a module is irregular. There exists an algorithm, due to Assi, Castro and Granger [2, which calculates, using non-commutative Gröbner basis, the slopes of a $\mathcal{D}$-module with respect to a hypersurface. In our work, we shall not use this algorithm because, although it is very useful in particular cases, it seems untreatable (so many Gröbner basis calculations) in general ones.

The aim of our study are the hypergeometric systems defined by one row. The hypergeometric systems, in general, are systems of partial differential equations with polynomial coefficients, so it defines a left ideal in the Weyl algebra. Given a $d \times n$ integer matrix $A$ with $\operatorname{rank}(A)=d$ and a complex vector $\beta \in \mathbb{C}^{d}$, let $H_{A}(\beta)$ denote the left ideal generated by

$$
\begin{gathered}
\partial^{u}-\partial^{v}, \quad u, v \in \mathbb{N}^{n} \quad \text { such that } \quad A u^{t}=A v^{t}, \\
\sum_{j=1}^{n} a_{i j} x_{j} \partial_{j}-\beta_{i}, \quad i=1, \ldots, d .
\end{gathered}
$$

The operators defined in (1.1) are called toric operators, and the ideal defined by them in the commutative subring $\mathbb{C}\left[\partial_{1}, \ldots, \partial_{n}\right]$ is called the toric ideal and it is usually denoted by $I_{A}$. We shall also use the notation $I_{A}$ to denote the extension of 
that ideal in the Weyl algebra or in $\mathcal{D}$. On the other hand, for the operators defined in (1.2), that are usually called homogeneity operators, we shall use the notation $\theta_{i}=x_{i} \partial_{i}$ and we represent those operators as the vector of operators $A \theta^{t}-\beta$.

The ideal $H_{A}(\beta) \subset \mathcal{A}_{n}$ is called the $A$-hypergeometric system with parameter $\beta$. These systems were introduced by Gel'fand, Kapranov and Zelevinski [4]. We usually denote by $\mathcal{H}_{A}(\beta)$ the module $\mathcal{A} / H_{A}(\beta)$. The module defined by $H_{A}(\beta)$ is holonomic; see 4 and 1 for the general case.

A result by Hotta [7] assures that if the toric ideal $I_{A}$ is homogeneous with respect to the usual grading, the hypergeometric system is regular. This condition, in the case of $A$ a $1 \times n$ matrix, is equivalent to $A$ being a multiple of the matrix $(1 \ldots 1)$.

In this paper, we treat the case when $A$ is a matrix defined by one row, and it does not verify this condition. The two main results are theorems 3.3 and 4.2 we find that, for matrices with at least three different elements the system always has a slope defined with respect to a hyperplane. We also calculate all the slopes with respect to the coordinate hyperplanes at the origin. The case with only two different elements was studied in [6]: in this case if the semigroup defined by the columns of $A$ is reduced, we have always a slope with respect to a hyperplane; when this semigroup is not reduced, we must do a change of coordinates and find a slope at infinity.

This work generalizes the paper [3] by Castro and Takayama which treats the case of hypergeometric systems defined by one row, with a matrix of the following form $\left(1 a_{2} \ldots a_{n}\right)$, with $1<a_{2}<\cdots<a_{n}$. In [3] they used a theorem of Laurent and Mebkhout [11] about restrictions and slopes. They need to compute the restriction of the hypergeometric system. In this process it is very useful that $a_{1}=1$. If not, the computation of the $b$-function (necessary in the known algorithms to compute restrictions) is very hard. On the other hand, to use the theorem it is also necessary that the calculation of the slopes in the new $\mathcal{D}$-module obtained must be easier to do, but if $a_{1} \neq 1$, it is possible that the presentation of the new module is in $\mathcal{D}^{r}$, with $r>1$. To generalize this paper we need to use different techniques.

\section{Filtrations AND SlOPes}

The rings of differential operators $\mathcal{A}$ and $\mathcal{D}$ admit several filtrations (the analogous to graduations in non-commutative rings). In this section we define the $F$-filtration or filtration by the order of the operator, the $V$-filtration or MalgrangeKashiwara filtration, and a family of filtrations defined as a combination of these. We give the definition of the filtrations with respect to the ring $\mathcal{D}$. Analogous definitions are valid in the Weyl algebra. With these objects we can formally define what a slope of a $\mathcal{D}$-module is.

Given an operator $P \in \mathcal{D}, P \neq 0$,

$$
P=\sum_{\beta} a_{\beta}(x) \partial^{\beta} .
$$

We define the $F$-order of this operator as

$$
\operatorname{ord}_{F}(P)=\max \left\{|\beta|, a_{\beta} \neq 0\right\}
$$

and $\operatorname{ord}_{F}(0)=-\infty$. 
For any $k$ we define the vector subspaces of the operators of order $k$ or less than $k$, and the operators of order less than $k$, and we denote them as

$$
\begin{gathered}
F_{k}(\mathcal{D})=\left\{P \in \mathcal{D}, \text { such that } \operatorname{ord}_{F}(P) \leq k\right\}, \\
F_{<k}(\mathcal{D})=\left\{P \in \mathcal{D}, \text { such that } \operatorname{ord}_{F}(P)<k\right\} .
\end{gathered}
$$

This allows us to define the graded algebra

$$
\operatorname{gr}^{F}(\mathcal{D})=\bigoplus_{k} \operatorname{gr}_{k}^{F}(\mathcal{D})=\bigoplus_{k} F_{k}(\mathcal{D}) / F_{<k}(\mathcal{D}) .
$$

For any operator $P$ we can define its $F$-symbol, an element in the graded ring, as

$$
\sigma_{F}(P)=\sum_{\beta,|\beta|=o r d_{F}(P)} a_{\beta}(x) \xi^{\beta} .
$$

Given an ideal $I$ in $\mathcal{D}$ we define the graded ideal

$$
\operatorname{gr}^{F}(I)=\left\langle\sigma_{F}(P), P \in I\right\rangle .
$$

In this case $\operatorname{gr}^{F}(\mathcal{D}) \cong \mathbb{C}\{x\}[\xi]$ and $\operatorname{gr}^{F}(\mathcal{A}) \cong \mathbb{C}[x, \xi]$.

In an analogous way we can define the $V$-filtration or Malgrange-Kashiwara filtration, which is defined with respect to a hypersurface. If we consider the hypersurface $x_{j}=0$, for a non-zero operator $P$,

$$
P=\sum_{\alpha, \beta} a_{\alpha, \beta} x^{\alpha} \partial^{\beta} .
$$

We define its $V$-order as

$$
\operatorname{ord}_{V}(P)=\max \left\{\beta_{j}-\alpha_{j}, a_{\alpha, \beta} \neq 0\right\},
$$

and $\operatorname{ord}_{V}(0)=-\infty$. The vector subspaces are defined as

$$
\begin{gathered}
V_{k}(\mathcal{D})=\left\{P \in \mathcal{D}, \text { such that } \operatorname{ord}_{V}(P) \leq k\right\}, \\
V_{<k}(\mathcal{D})=\left\{P \in \mathcal{D}, \text { such that } \operatorname{ord}_{V}(P)<k\right\} .
\end{gathered}
$$

Then, the $V$-graded ring is

$$
\operatorname{gr}^{V}(\mathcal{D})=\bigoplus_{k} \operatorname{gr}_{k}^{V}(\mathcal{D})=\bigoplus_{k} V_{k}(\mathcal{D}) / V_{<k}(\mathcal{D}) .
$$

For an operator we can define its $V$-symbol as

$$
\sigma_{V}(P)=\sum_{(\alpha, \beta), \beta_{j}-\alpha_{j}=o r d_{V}(P)} a_{\alpha, \beta} x^{\alpha} \xi^{\beta} .
$$

As before we can define the graded ideal. The great difference in this case is that the graded ring is not commutative as before. Indeed

$$
\operatorname{gr}^{V}(\mathcal{D}) \cong \mathbb{C}\left[x_{j}\right]\left\{x_{1}, \ldots, x_{j-1}, x_{j+1}, \ldots, x_{n}\right\}\langle\partial\rangle
$$

with the usual relations $\left[x_{i}, x_{j}\right]=0,\left[\partial_{i}, \partial_{j}\right]=0$, and $\left[\partial_{i}, x_{j}\right]=\delta_{i, j}$. For the Weyl algebra, $\operatorname{gr}^{V}(\mathcal{A}) \cong \mathcal{A}$.

Using the above filtrations $F$ and $V$ (with respect to $x_{j}=0$ ), we can define an ordered family of filtrations. Given $(p, q) \neq(0,0)$, non-negative integers, we define the linear form over $\mathbb{Q}^{2}$, given by $L(a, b)=p a+q b$. Then, given an operator as before, we define the $L$-order of a non-zero operator:

$$
\operatorname{ord}_{L}(P)=\max \left\{L\left(|\beta|, \beta_{j}-\alpha_{j}\right), a_{\alpha, \beta} \neq 0\right\},
$$


as usual $\operatorname{ord}_{L}(0)=-\infty$. We also define the vector subspaces:

$$
\begin{gathered}
L_{k}(\mathcal{D})=\left\{P \in \mathcal{D}, \text { such that } \operatorname{ord}_{L}(P) \leq k\right\}, \\
L_{<k}(\mathcal{D})=\left\{P \in \mathcal{D}, \text { such that } \operatorname{ord}_{L}(P)<k\right\} .
\end{gathered}
$$

The $L$-symbol of an operator is defined as

$$
\sigma_{L}(P)=\sum_{(\alpha, \beta), L\left(|\beta|, \beta_{j}-\alpha_{j}\right)=\operatorname{ord}_{L}(P)} a_{\alpha, \beta} x^{\alpha} \xi^{\beta} .
$$

As before, we define the $L$-graded ideal and the graded $\operatorname{ring} \operatorname{gr}^{L}(\mathcal{D})$. If $p \neq 0$, then $\operatorname{gr}^{L}(\mathcal{D}) \cong \mathbb{C}\{x\}[\partial]$ and $\operatorname{gr}^{L}(\mathcal{A}) \cong \mathbb{C}[x, \partial]$.

We can always consider the $F$-filtration as an $L$-filtration for $(p, q)=(1,0)$, and also the $V$-filtration as the $L$-filtration for $(p, q)=(0,1)$. In general, to simplify notation, we shall write $L=p F+q V$ to note the filtration $L$ defined as before.

We can define an order among the $L$-filtrations. Given two $L$-filtrations, $L_{1}$ defined by $(p, q)$ and $L_{2}$ by $\left(p^{\prime}, q^{\prime}\right), L_{1}<L_{2}$ if $-p / q<-p^{\prime} / q^{\prime}$. Given a $L$-filtration we define its slope as the ratio $-p / q$.

Definition 2.1. Given a left ideal $I$ and an $L$-filtration $L \neq V$, we define the $L$-characteristic variety, denoted $C h^{L}(\mathcal{D} / I)$, as the analytic variety of $\mathbb{C}^{2 n}$ defined by its graded ideal $\operatorname{gr}^{L}(I)$, i.e.,

$$
C h^{L}(\mathcal{D} / I)=\left\{(x, \xi) \in \mathbb{C}^{2 n} \mid \sigma^{L}(P)(x, \xi)=0, \quad \text { for all } \quad P \in I\right\} .
$$

If the left ideal $I$ is in the Weyl algebra we define similarly the $L$-characteristic variety.

Definition $2.2(9])$. Let $I$ be an ideal of $\mathcal{D}$. The slopes of the $\mathcal{D}$-module $\mathcal{D} / I$ with respect to $x_{j}=0$ are the slopes of the linear forms $L \neq F, V$ (with $V$ defined with respect to $x_{j}=0$ ) such that $\sqrt{\operatorname{gr}^{L}(I)}$ is not bihomogeneous for the $F$ and $V$ filtrations.

In the Weyl algebra, we can define a filtration for a given vector $(u, v) \in \mathbb{R}^{2 n}$ such that $u_{i}+v_{i} \geq 0$ :

$$
\operatorname{ord}_{(u, v)}(P)=\max \left\{\alpha u+\beta v, \quad a_{\alpha, \beta} \neq 0\right\},
$$

$W_{k}=\{P \in \mathcal{A}$ such that $\alpha u+\beta v \leq k\}, \quad W_{<k}=\{P \in \mathcal{A}$ such that $\alpha u+\beta v<k\}$,

$$
\begin{gathered}
\operatorname{gr}^{(u, v)}(\mathcal{A})=\bigoplus_{k} \operatorname{gr}_{k}^{W}(\mathcal{A})=\bigoplus_{k} W_{k}(\mathcal{A}) / W_{<k}(\mathcal{A}), \\
\operatorname{in}_{(u, v)}(P)=\sum_{(\alpha, \beta) \alpha u+\beta v=\operatorname{ord}_{(u, v)}(P)} a_{\alpha, \beta} x^{\alpha} \xi^{\beta} .
\end{gathered}
$$

For this weight vector we shall use the notation of initial ideals:

$$
\operatorname{in}_{(u, v)}(I)=\left\langle\operatorname{in}_{(u, v)}(P), P \in I\right\rangle .
$$

It is clear that, if we consider the filtration $L=p F+q V$, defined with respect to the hyperplane $x_{i}=0$, then

$$
\operatorname{gr}^{L}(I)=\operatorname{in}_{(0, \ldots, 0,-q, 0, \ldots, 0, p, \ldots, p, p+q, p, \ldots, p)}(I)
$$

with $-q$ in the $i$-th coordinate and $p+q$ in the $i+n$-th coordinate. 


\section{HypergeOMETRIC SYSTEMS DEFINED BY A REDUCED SEMIGROUP}

In this section, we consider a $1 \times n$ integer matrix $A=\left(a_{1} a_{2} \ldots a_{n}\right)$, such that the semigroup defined by its elements is reduced or, equivalently, such that all the elements $a_{i}$ are positive.

First of all we introduce some notation and a theorem from [14]. Here we do not consider that the ideal $I_{A}$ is homogeneous: the result is true without this hypothesis.

Definition 3.1 ([14]). Given a $d \times n$ integer matrix $A$ and a complex vector $\beta \in \mathbb{C}^{d}$, we define the $(u, v)$-fake initial ideal, when $u_{i}+v_{i} \geq 0$, as

$$
\operatorname{fin}_{(u, v)}\left(H_{A}(\beta)\right)=\operatorname{in}_{v}\left(I_{A}\right)+\left\langle\operatorname{in}_{(u, v)}\left(A \theta^{t}-\beta\right)\right\rangle,
$$

where $\operatorname{in}_{(u, v)}(f)$ denotes the initial terms of $f$, or the initial ideal, with respect to the weight vector $(u, v)$.

In general we have the following result:

Theorem 3.2 (Thm. 4.3.5 of [14]). Given a $d \times n$ integer matrix $A$ and a complex vector $\beta \in \mathbb{C}^{d}$, for a fixed weight vector $(u, v)$, such that $u_{i}+v_{i}>0$, if $\operatorname{in}_{(u, v)}\left(A \theta^{t}-\beta\right)$ forms a regular sequence over the ring $\operatorname{gr}^{(u, v)}(\mathcal{A}) / \operatorname{in}_{v}\left(I_{A}\right)$, then

$$
\operatorname{in}_{(u, v)}\left(H_{A}(\beta)\right)=\operatorname{fin}_{(u, v)}\left(H_{A}(\beta)\right) .
$$

Proof. We consider as in 14 the following exact sequence of modules over the algebra $\operatorname{gr}^{(u, v)}(\mathcal{A}) / \operatorname{in}_{v}\left(I_{A}\right)$ :

$\bigoplus_{i=1}^{d}\left(\operatorname{gr}^{(u, v)}(\mathcal{A}) / \operatorname{in}_{v}\left(I_{A}\right)\right) \cdot e_{i} \stackrel{\bar{d}_{1}}{\rightarrow} \operatorname{gr}^{(u, v)}(\mathcal{A}) / \operatorname{in}_{v}\left(I_{A}\right) \rightarrow \operatorname{gr}^{(u, v)}(\mathcal{A}) / \operatorname{fin}_{(u, v)}\left(H_{A}(\beta)\right) \rightarrow 0$ where $\bar{d}_{1}\left(\sum_{i=1}^{n} P_{i} e_{i}\right)=\sum_{i=1}^{n} P_{i} \operatorname{in}_{(u, v)}\left(\left(A \theta^{t}-\beta\right)_{i}\right)$.

Since $\operatorname{in}_{(u, v)}\left(\left(A \theta^{t}-\beta\right)_{j}\right) e_{i}-\operatorname{in}_{(u, v)}\left(\left(A \theta^{t}-\beta\right)_{i}\right) e_{j}$ clearly belongs to the kernel of $\bar{d}_{1}$, we can extend the exact sequence to the Koszul complex $K_{\bullet}^{\beta}\left(\operatorname{gr}^{(u, v)}\left(\mathcal{A} / I_{A}\right)\right)$ :

$$
\cdots \stackrel{\bar{d}_{2}}{\longrightarrow} K_{1}^{\beta}\left(\operatorname{gr}^{(u, v)}\left(\mathcal{A} / I_{A}\right)\right) \stackrel{\bar{d}_{1}}{\longrightarrow} K_{0}^{\beta}\left(\operatorname{gr}^{(u, v)}\left(\mathcal{A} / I_{A}\right)\right) \longrightarrow 0
$$

where

$$
K_{p}^{\beta}\left(\operatorname{gr}^{(u, v)}\left(\mathcal{A} / I_{A}\right)\right)=\bigoplus_{1 \leq i_{1}<\cdots<i_{p} \leq n} \operatorname{gr}^{(u, v)}\left(\mathcal{A} / I_{A}\right) e_{i_{1} \cdots i_{p}}
$$

and

$$
\bar{d}_{p}\left(e_{i_{1} \cdots i_{p}}\right)=\sum_{r=1}^{p}(-1)^{r-1} \operatorname{in}_{(u, v)}\left(\left(A \theta^{t}-\beta\right)_{i_{r}}\right) e_{i_{1} \cdots \hat{i_{r}} \cdots i_{p}} .
$$

We can also define the Koszul complex (with non-homogeneous elements) $K_{\bullet}^{\beta}\left(\mathcal{A} / I_{A}\right)$ as

$$
\cdots \longrightarrow K_{2}^{\beta}\left(\mathcal{A} / I_{A}\right) \stackrel{d_{2}}{\longrightarrow} K_{1}^{\beta}\left(\mathcal{A} / I_{A}\right) \stackrel{d_{1}}{\longrightarrow} K_{0}^{\beta}\left(\mathcal{A} / I_{A}\right) \longrightarrow 0,
$$

where

$$
K_{p}^{\beta}\left(\mathcal{A} / I_{A}\right)=\bigoplus_{1 \leq i_{1}<\cdots<i_{p} \leq n} \mathcal{A} / I_{A} e_{i_{1} \cdots i_{p}}
$$

and

$$
d_{p}\left(e_{i_{1} \cdots i_{p}}\right)=\sum_{r=1}^{p}(-1)^{r-1}\left(\left(A \theta^{t}-\beta\right)_{i_{r}}\right) e_{i_{1} \cdots \widehat{i_{r} \cdots i_{p}}}
$$


This complex can be filtered by

$$
F_{q}\left(K_{p}^{\beta}\left(\mathcal{A} / I_{A}\right)\right)=\bigoplus_{1 \leq i_{1}<\cdots<i_{p} \leq n} F_{q-\sum_{k=1}^{p} c_{i_{k}}} \mathcal{A} / I_{A} e_{i_{1} \cdots i_{p}},
$$

where $c_{i_{k}}=\operatorname{ord}_{(u, v)}\left(\left(A \theta^{t}-\beta\right)_{i_{k}}\right)$.

Clearly

$$
K_{\bullet}^{\beta}\left(\operatorname{gr}^{(u, v)}\left(\mathcal{A} / I_{A}\right)\right)=\operatorname{gr}^{(u, v)}\left(K_{\bullet}^{\beta}\left(\mathcal{A} / I_{A}\right)\right) .
$$

Using this filtration it is easy to see that the sequence

$$
K_{1}^{\beta}\left(\operatorname{gr}^{(u, v)}\left(\mathcal{A} / I_{A}\right)\right) \longrightarrow K_{0}^{\beta}\left(\operatorname{gr}^{(u, v)}\left(\mathcal{A} / I_{A}\right)\right) \longrightarrow \operatorname{gr}^{(u, v)}\left(\mathcal{H}_{A}(\beta)\right) \longrightarrow 0
$$

is exact if $H_{1}\left(K_{\bullet}^{\beta}\left(\operatorname{gr}^{(u, v)}\left(\mathcal{A} / I_{A}\right)\right)\right)=0$.

If this sequence is exact, we have proved the statement, so all we need to prove is that $H_{1}\left(K_{\bullet}^{\beta}\left(\operatorname{gr}^{(u, v)}\left(\mathcal{A} / I_{A}\right)\right)\right)=0$, or it is enough to see that the elements in $\left\{\operatorname{in}_{(u, v)}\left(\left(A \theta^{t}-\beta\right)_{1}\right), \ldots, \operatorname{in}_{(u, v)}\left(\left(A \theta^{t}-\beta\right)_{d}\right)\right\}$ form a regular sequence in the commutative graded ring $\operatorname{gr}^{(u, v)}(\mathcal{A}) / \mathrm{in}_{v}\left(I_{A}\right)$.

First, we consider $a_{1}<a_{2}<\cdots<a_{n}$, and we study a generalization of the case considered in [3], where the condition $a_{1}=1$ was added.

Theorem 3.3. Given $A, a 1 \times n$ matrix, and $\beta \in \mathbb{C}$, such that $A=\left(a_{1} a_{2} \ldots a_{n}\right)$, with $0<a_{1}<a_{2}<\cdots<a_{n}$, the hypergeometric system $H_{A}(\beta)$ has no slopes with respect to the hyperplanes $x_{i}=0, i<n$, and has only the slope $\frac{a_{n-1}}{a_{n-1}-a_{n}}$ with respect to $x_{n}=0$. The singular locus of the system is $\left\{x_{n}=0\right\}$.

(i) If we consider a filtration $L=p F+q V, p>0$, with $V$ defined with respect to $x_{i}=0, i<n$, then

$$
C h^{L}\left(\mathcal{H}_{A}(\beta)\right)=\mathcal{V}\left(\xi_{1}, \ldots, \xi_{n-1}, x_{n} \xi_{n}\right) .
$$

(ii) If we consider a filtration $L=p F+q V, p>0$, with $V$ defined with respect to $x_{n}=0$, with $L<a_{n-1} F+\left(a_{n}-a_{n-1}\right) V$, then

$$
C h^{L}\left(\mathcal{H}_{A}(\beta)\right)=\mathcal{V}\left(\xi_{1}, \ldots, \xi_{n-1}, x_{n} \xi_{n}\right) .
$$

(iii) If $L>a_{n-1} F+\left(a_{n}-a_{n-1}\right) V$, then

$$
C h^{L}\left(\mathcal{H}_{A}(\beta)\right)=\mathcal{V}\left(\xi_{1}, \ldots, \xi_{n-2}, \xi_{n}, x_{n-1} \xi_{n-1}\right) .
$$

Proof. In first place we shall prove the first part of the theorem. Given $A=$ $\left(a_{1} a_{2} \ldots a_{n}\right)$, we consider the set of elements in the toric ideal $I_{A}$ :

$$
C_{i, j}=\partial_{i}^{a_{j}}-\partial_{j}^{a_{i}}, \quad i<j .
$$

First of all, we prove that if we consider the hyperplane $x_{j}=0$ with $j<n$, there are not slopes of the system with respect to this hypersurface. Let $L$ be a filtration defined with respect to $x_{j}=0$, such that $L=p F+q V$, with $p \neq 0$. We consider the operators $C_{k, n}$. If $k=j$, then $\sigma_{F}\left(C_{j, n}\right)=\xi_{j}^{a_{n}}$, so it is clear that $\sigma_{L}\left(C_{j, n}\right)=\xi_{j}^{a_{n}}$. If $k \neq j$, we have that $\sigma_{L}\left(C_{k, n}\right)=\sigma_{F}\left(C_{k, n}\right)=\xi_{k}^{a_{n}}$. Finally,

$$
\left\{\xi_{1}^{a_{n}}, \xi_{2}^{a_{n}}, \ldots, \xi_{n-1}^{a_{n}}\right\} \subset \operatorname{gr}^{L}\left(H_{A}(\beta)\right) \Rightarrow\left\{\xi_{1}, \xi_{2}, \ldots, \xi_{n-1}\right\} \subset \sqrt{\operatorname{gr}^{L}\left(H_{A}(\beta)\right)} .
$$

Now, if we consider the element $Q=a_{1} x_{1} \partial_{1}+a_{2} x_{2} \partial_{2}+\cdots+a_{n} x_{n} \partial_{n}-\beta$, we have

$$
\sigma_{L}(Q)=a_{1} x_{1} \xi_{1}+a_{2} x_{2} \xi_{2}+\cdots+a_{n} x_{n} \xi_{n} \in \operatorname{gr}^{L}\left(H_{A}(\beta)\right),
$$


SO

$$
\left\{\xi_{1}, \xi_{2}, \ldots, \xi_{n-1}, x_{n} \xi_{n}\right\} \subset \sqrt{\operatorname{gr}^{L}\left(H_{A}(\beta)\right)},
$$

and $L$ does not define a slope of the system. This fact comes from the property of the module $\mathcal{H}_{A}(\beta)$ being holonomic; see [1]. So for $L \neq V$ we have that $\operatorname{gr}^{L}\left(\mathcal{H}_{A}(\beta)\right)$ is holonomic too, and then the $L$-characteristic variety can be decomposed in irreducible components, all of dimension $n$, and each of those components is included in some of those defined before by $\left\{\xi_{1}, \xi_{2}, \ldots, \xi_{n-1}, x_{n} \xi_{n}\right\}$. But all these components are bihomogenous, hence $L$ does not define a slope. For further details see [5].

We prove now, at the same time, parts (i) and (ii). We fix the hyperplane $x_{n}=0$. We choose a filtration $L$ defined as $L=p F+q V$, such that $L<a_{n-1} F+$ $\left(a_{n}-a_{n-1}\right) V$ (the same results are true in the (i) case). We have that $\sigma_{L}\left(C_{i, n}\right)=$ $\xi_{i}^{a_{n}}$, and the operators $\left\{C_{1, n}, C_{2, n}, \ldots, C_{n-1, n}\right\}$ are a $\mathbb{Q}$-basis of $\operatorname{ker}(A)$.

We are interested in the toric ideal, $I_{A}$. Due to the fact that all $a_{i}>0$, we have that the toric ideal $I_{A}$ is homogeneous with respect to the grading defined by $A$. If we note $\widehat{I_{A}}$ the ideal generated by $C_{i, n}$, then it is also graded, and $\widehat{I_{A}} \subset I_{A}$.

To compute a set of generators of $I_{A}$ we use the ideal quotients. Using lemma 12.1 of [15] we can obtain $\left(\widehat{I_{A}}: \partial_{i}^{\infty}\right)$. First of all, we calculate $\left(\widehat{I_{A}}: \partial_{n}^{\infty}\right)$ so that, choosing the monomial order $\prec$, graded with respect to this grading such that $\partial_{1} \succ \partial_{2} \succ \cdots \succ \partial_{n}$, the set $\left\{C_{1, n}, \ldots, C_{n-1, n}\right\}$ forms a Gröbner basis (with respect to such a monomial ordering) of $\widehat{I_{A}}$. In the same way, if we fix an $i<n$, we can consider the new monomial ordering $\prec^{\prime}$, defined as the graded monomial ordering such that $\partial_{1} \succ^{\prime} \partial_{2} \succ^{\prime} \cdots \succ^{\prime} \partial_{i-1} \succ^{\prime} \partial_{i+1} \succ^{\prime} \cdots \succ^{\prime} \partial_{n} \succ^{\prime} \partial_{i}$. So, finally we obtain

$$
\left(\left\langle C_{1, n}, C_{2, n}, \ldots, C_{n-1, n}\right\rangle:\left(\partial_{1} \cdots \partial_{n}\right)^{\infty}\right)=\left\langle C_{1, n}, C_{2, n}, \ldots, C_{n-1, n}\right\rangle .
$$

Both ideals $I_{A}$ and $\widehat{I_{A}}$ are saturated, and $\widehat{I_{A}} \subset I_{A}$. We have defined $\widehat{I_{A}}$ from a $\mathbb{Q}$-basis of $\operatorname{ker}(A)$, hence we have that for any $\partial^{\mathbf{u}^{+}}-\partial^{\mathbf{u}^{-}} \in I_{A}$ there always exists an element $\partial^{\mathbf{v}^{+}}-\partial^{\mathbf{v}^{-}} \in \widehat{I_{A}}$, and an $a \in \mathbb{N}$ such that $a \mathbf{u}=\mathbf{v}$.

On the other hand, it is clear that

$$
\left\{\xi_{1}^{a_{n}}, \xi_{2}^{a_{n}}, \ldots, \xi_{n-1}^{a_{n}}\right\}
$$

forms a regular sequence in $\mathbb{C}[\xi]$, so using proposition 4.3 .2 of [14] we have that

$$
\left\langle\xi_{1}^{a_{n}}, \xi_{2}^{a_{n}}, \ldots, \xi_{n-1}^{a_{n}}\right\rangle=\operatorname{in}_{(p, \ldots, p, p+q)}\left(\widehat{I_{A}}\right) .
$$

This implies, with the above properties,

$$
\begin{gathered}
\left\langle\xi_{1}^{a_{n}}, \xi_{2}^{a_{n}}, \ldots, \xi_{n-1}^{a_{n}}\right\rangle=\operatorname{in}_{(p, \ldots, p, p+q)}\left(\widehat{I_{A}}\right) \subset \operatorname{in}_{(p, \ldots, p, p+q)}\left(I_{A}\right) \\
\subset\left\langle\xi_{1}, \xi_{2}, \ldots, \xi_{n-1}\right\rangle=\sqrt{\operatorname{in}_{(p, \ldots, p, p+q)}\left(\widehat{I_{A}}\right)}=\sqrt{\operatorname{in}_{(p, \ldots, p, p+q)}\left(I_{A}\right)} .
\end{gathered}
$$

We want to prove that $\sigma_{L}\left(a_{1} x_{1} \partial_{1}+\cdots+a_{n} x_{n} \partial_{n}-\beta\right)$ forms a regular sequence over $\mathbb{C}[x, \xi] / \mathrm{gr}^{L}\left(I_{A}\right)$, that is, this element is not a zero divisor in this ring. But, if we prove that this element is not a zero divisor over $\mathbb{C}[x, \xi] / \operatorname{gr}^{L}\left(\widehat{I_{A}}\right)$, we obtain the desired result.

The ring $\mathbb{C}[x, \xi] / \operatorname{gr}^{L}\left(\widehat{I_{A}}\right)$ is a graded ring; we must see that $\sigma_{L}(A \theta-\beta)$ is not a zero divisor, that is, if $h \sigma_{L}(A \theta-\beta) \in \operatorname{gr}^{L}\left(\widehat{I_{A}}\right)$, then $h \in \operatorname{gr}^{L}\left(\widehat{I_{A}}\right) \cdot \operatorname{But}^{\operatorname{gr}^{L}}\left(\widehat{I_{A}}\right)$ is a monomial graded ideal, so all the monomials in $h x_{n} \xi_{n} \in \operatorname{gr}^{L}\left(\widehat{I_{A}}\right)$, thus $h \in \operatorname{gr}^{L}\left(\widehat{I_{A}}\right)$. 
So, we have proved that, for any $L$ filtration, with $L<a_{n-1} F+\left(a_{n}-a_{n-1}\right) V$,

$$
\begin{gathered}
\operatorname{gr}^{L}\left(H_{A}(\beta)\right)=\operatorname{fin}_{(0, \ldots, 0,-q, p, \ldots, p, p+q)}\left(H_{A}(\beta)\right) \\
=\operatorname{in}_{(p, \ldots, p, p+q)}\left(I_{A}\right)+\sigma_{L}\left(a_{1} x_{1} \partial_{1}+\cdots+a_{n} x_{n} \partial_{n}-\beta\right)
\end{gathered}
$$

and it is clear that this is also true for any filtration $L$ defined with respect to the Malgrange-Kashiwara filtration with respect to a hypersurface $x_{i}=0$ with $i<n$.

In particular we have that

$$
\begin{gathered}
C h^{L}\left(H_{A}(\beta)\right)=\mathcal{V}\left(\sqrt{\operatorname{fin}_{(0, \ldots, 0,-q, p, \ldots, p, p+q)}\left(H_{A}(\beta)\right.}\right) \\
=\mathcal{V}\left(\sqrt{\sqrt{\operatorname{in}_{(p, \ldots, p, p+q)}\left(I_{A}\right)}+\sqrt{\sigma_{L}\left(a_{1} x_{1} \partial_{1}+\cdots+a_{n} x_{n} \partial_{n}-\beta\right)}}\right) \\
=\mathcal{V}\left(\xi_{1}, \ldots, \xi_{n-1}, x_{n} \xi_{n}\right) .
\end{gathered}
$$

Finally, we prove part (iii). We now consider a new filtration $L$ defined with respect the Malgrange-Kashiwara filtration, with respect to the hypersurface $x_{n}=$ 0 , such that $L>a_{n-1} F+\left(a_{n}-a_{n-1}\right) V$. In this case, we obtain that

$$
\operatorname{in}_{(p, \ldots, p, p+q)}\left(\widehat{I_{A}}\right)=\left\langle\xi_{1}^{a_{n}}, \xi_{2}^{a_{n}}, \ldots, \xi_{n-2}^{a_{n}}, \xi_{n}^{a_{n-1}}\right\rangle .
$$

Using the same argument as before, we have that $\sigma_{L}\left(a_{1} x_{1} \partial_{1}+\cdots+a_{n} x_{n} \partial_{n}-\beta\right)$ is also a regular sequence over $\mathbb{C}[x, \xi] / \operatorname{in}_{(p, \ldots, p, p+q)}\left(I_{A}\right)$, so

$$
\begin{gathered}
\operatorname{gr}^{L}\left(H_{A}(\beta)\right)=\operatorname{fin}_{(0, \ldots, 0,-q, p, \ldots, p, p+q)}\left(H_{A}(\beta)\right) \\
=\operatorname{in}_{(p, \ldots, p, p+q)}\left(I_{A}\right)+\sigma_{L}\left(a_{1} x_{1} \partial_{1}+\cdots+a_{n} x_{n} \partial_{n}-\beta\right) .
\end{gathered}
$$

We want to calculate the $L$-characteristic variety as before. In this case,

$$
C h^{L}\left(H_{A}(\beta)\right)=\mathcal{V}\left(\xi_{1}, \xi_{2}, \ldots, \xi_{n-2}, \xi_{n}, x_{n-1} \xi_{n-1}\right)
$$

This change in the characteristic variety tells us that $L=a_{n-1} F+\left(a_{n}-a_{n-1}\right) V$ is a slope of the hypergeometric system. It is also clear, as before, that any other filtration $L$ is not a slope of our system.

In theorem 3.3, we had the hypothesis $a_{1}<a_{2}<\cdots<a_{n}$. We now describe the general case.

Given a $1 \times n$ integer matrix, which defines a reduced semigroup, we can always order its elements. We consider, in the first place, that one of the elements in the matrix is repeated, and the repeated element is not the largest one. To simplify notation, we suppose that $a_{1}$ is the repeated element, and it is written twice. It is clear that it can be easily generalized. So,

$$
A=\left(\begin{array}{llllll}
a_{1} & a_{1} & a_{2} & a_{3} & \cdots & a_{n-1}
\end{array}\right) \quad a_{1}<a_{2}<\cdots<a_{n-1} .
$$

If we consider the following elements in the toric ideal:

$$
C_{1, n}=\partial_{1}^{a_{n-1}}-\partial_{n}^{a_{1}}, \quad C_{i, n}=\partial_{i}^{a_{n-1}}-\partial_{n}^{a_{i-1}}, \quad 2 \leq i \leq n-1,
$$

they form a $\mathbb{Q}$ basis of $\operatorname{ker}(A)$. Repeating the process in the proof of theorem 3.3 we obtain that the $L$-symbol of the homogeneity operator forms a regular sequence, for every filtration $L \neq a_{n-2} F+\left(a_{n-1}-a_{n-2}\right) V$. We see that theorem 3.3 can be rewritten in this case.

On the other hand, if the repeated element is the largest one, and we suppose that it is written twice, we have

$$
A=\left(\begin{array}{lllll}
a_{1} & a_{2} & \ldots & a_{n-1} & a_{n-1}
\end{array}\right), \quad a_{1}<a_{2}<\cdots<a_{n-1} .
$$


If we do the following change of coordinates:

$$
x_{1}=x_{1}^{\prime} x_{2}=x_{2}^{\prime} \cdots x_{n-1}=x_{n-1}^{\prime} x_{n}^{\prime}=x_{n-1}+x_{n}
$$

we obtain

$$
\partial_{1}=\partial_{1}^{\prime} \partial_{2}=\partial_{2}^{\prime} \cdots \partial_{n-1}=\partial_{n-1}^{\prime}+\partial_{n}^{\prime} \partial_{n}=\partial_{n}^{\prime} .
$$

If we calculate the slopes of the new system obtained with this change of coordinates, we are studying the slopes of our system with respect to $x_{1}=0, x_{2}=0, \ldots$, $x_{n-1}=0$ or $x_{n-1}+x_{n}=0$.

If we do the change of coordinates we obtain the new left ideal generated by

$$
\begin{gathered}
C_{1, n}=\partial_{1}^{\prime a_{n-1}}-\partial_{n}^{\prime a_{1}}, \ldots, C_{n-2, n-1}=\partial_{n-2}^{\prime}{ }^{a_{n-1}}-\partial_{n}^{\prime a_{n-2}}, C_{n-1, n}=\partial_{n-1}^{\prime} \\
a_{1} x_{1}^{\prime} \partial_{1}^{\prime}+a_{2} x_{2}^{\prime} \partial_{2}^{\prime}+\cdots+a_{n-1} x_{n-1}^{\prime}\left(\partial_{n-1}^{\prime}+\partial_{n}^{\prime}\right)+a_{n-1}\left(x_{n}^{\prime}-x_{n-1}^{\prime}\right) \partial_{n}^{\prime}-\beta \\
=a_{1} x_{1}^{\prime} \partial_{1}^{\prime}+a_{2} x_{2}^{\prime} \partial_{2}^{\prime}+\cdots+a_{n-1} x_{n-1}^{\prime} \partial_{n-1}^{\prime}+a_{n-1} x_{n}^{\prime} \partial_{n}^{\prime}-\beta .
\end{gathered}
$$

Then the left ideal we have obtained is the same as

$$
\left.\left\langle\partial_{n-1}^{\prime}, H_{\left(a_{1}\right.} a_{2} \cdots a_{n-2} a_{n-1}\right)(\beta)\right\rangle,
$$

if we consider the names of the variables for the Gelfand'-Kapranov-Zelevinski system equal to $x_{1}^{\prime}, x_{2}^{\prime}, \ldots, x_{n-2}^{\prime}, x_{n}^{\prime}$.

This case has been solved in theorem 3.3. We do not have any slope with respect to the hyperplanes $x_{i}^{\prime}=0$ for $i<n$ and we have only one slope for the hyperplane $x_{n}^{\prime}=0$, the slope defined by the filtration $L=a_{n-2} F+\left(a_{n-1}-a_{n-2}\right) V$. As we said before, we have obtained one slope of our system, with respect to the hyperplane $x_{n-1}+x_{n}=0$.

\section{Hypergeometric Systems With NO REDUCED SEMigRoup}

In this section we shall study hypergeometric systems defined by an integer $1 \times n$ matrix $A$ such that the semigroup $S$ defined by its columns is not a reduced one. In other words $S \cap(-S)=0$ or, equivalently, the matrix $A$ has positive and negative elements. The case of an integer matrix with all of its elements negative is the same as the one with all the elements positive, because if we multiply the $d \times n$ matrix $A$ by a matrix $B$, such that $B \in G L_{d}(\mathbb{Q})$, and $B A$ is an integer matrix too, we have that $H_{A}(\beta)=H_{B A}(B \beta)$.

We suppose that $A$ has at least three different elements, because the case with only two elements was studied in 6 . It is precisely this case, a $1 \times 2$ matrix with one positive element and one negative, when there is no slope with respect to the coordinate hyperplanes, and we must look for the slopes at infinity.

In first place, let us consider $A$ with one negative element $-b_{1}$ and the other elements positive, different and ordered.

Lemma 4.1. Given $a 1 \times n$ matrix $A$ and $\beta \in \mathbb{C}$, such that $A=\left(-b_{1} a_{1} a_{2} \ldots a_{n-1}\right)$, with $b_{1}>0$ and $0<a_{1}<a_{2}<\cdots<a_{n-1}$, the hypergeometric system $H_{A}(\beta)$ has no slopes with respect to the hyperplanes $x_{i}=0, i<n$, and it has only the slope $\frac{a_{n-2}}{a_{n-2}-a_{n-1}}$ with respect to $x_{n}=0$. The singular locus of the system is $\left\{x_{1}=x_{n}=\right.$ $0\}$.

(i) If we consider a filtration $L=p F+q V, p>0$, with $V$ defined with respect to $x_{i}=0, i<n$, then

$$
C h^{L}\left(\mathcal{H}_{A}(\beta)\right)=\mathcal{V}\left(\xi_{1} \xi_{n}, \xi_{2}, \ldots, \xi_{n-1}, x_{1} \xi_{1}, x_{n} \xi_{n}\right) .
$$


(ii) If we consider a filtration $L=p F+q V, p>0$, with $V$ defined with respect to $x_{n}=0$, with $L<a_{n-2} F+\left(a_{n-1}-a_{n-2}\right) V$, then

$$
C h^{L}\left(\mathcal{H}_{A}(\beta)\right)=\mathcal{V}\left(\xi_{1} \xi_{n}, \xi_{2}, \ldots, \xi_{n-1}, x_{1} \xi_{1}, x_{n} \xi_{n}\right) .
$$

(iii) If $L>a_{n-2} F+\left(a_{n-1}-a_{n-2}\right) V$, then

$$
C h^{L}\left(\mathcal{H}_{A}(\beta)\right)=\mathcal{V}\left(\xi_{1} \xi_{n-1}, \xi_{2}, \ldots, \xi_{n-2}, \xi_{n}, x_{1} \xi_{1}, x_{n-1} \xi_{n-1}\right) .
$$

Proof. We shall use very similar arguments to those in theorem 3.3 . We consider the following elements in the toric ideal:

$$
C_{1, n}=\partial_{1}^{a_{n-1}} \partial_{n}^{b_{1}}-1, C_{2, n}=\partial_{2}^{a_{n-1}}-\partial_{n}^{a_{1}}, \ldots, C_{n-1, n}=\partial_{n-1}^{a_{n-1}}-\partial_{n}^{a_{n-2}} .
$$

Those elements form a $\mathbb{Q}$-basis of $\operatorname{ker}(A)$, and due to the fact of the semigroup not being reduced, as we have added to our set an element of the form $\partial^{u}-1$, if they form a $\mathbb{Z}$-basis, they generate the toric ideal. Let $\widehat{I_{A}}$ denote the ideal generated by $C_{1, n}, C_{2, n}, \ldots, C_{n-1, n}$. Then, for any element in the toric ideal $I_{A}, \partial^{\mathbf{u}^{+}}-\partial^{\mathbf{u}^{-}}$, there always exist $a \in \mathbb{N}$ and $\mathbf{v}=\mathbf{v}^{+}-\mathbf{v}^{-}$such that $\partial^{\mathbf{v}^{+}}-\partial^{\mathbf{v}^{-}} \in \widehat{I_{A}}$ and $\mathbf{v}=a \mathbf{u}$. Let $L=p F+q V$ be a filtration defined by any hyperplane $x_{i}=0$ with $i<n$. The set

$$
\left\{\sigma_{L}\left(C_{1,2}\right)=\xi_{1}^{a_{n-1}} \xi_{n}^{b_{1}}, \sigma_{L}\left(C_{2, n}\right)=\xi_{2}^{a_{n-1}}, \ldots, \sigma_{L}\left(C_{n-1, n}\right)=\xi_{n-1}^{a_{n-1}}\right\}
$$

forms a regular sequence, therefore they generate the ideal $\operatorname{in}_{(p, \ldots, p, p+q, p, \ldots, p)}\left(\widehat{I_{A}}\right)$.

We want to prove that the $L$-symbol of the homogeneity operator forms a regular sequence over $\mathbb{C}[x, \xi] / \operatorname{in}_{(p, \ldots, p, p+q, p, \ldots, p)}\left(I_{A}\right)$. If we prove that the $L$-symbol of the homogeneity operator forms a regular sequence over $\mathbb{C}[x, \xi] / \operatorname{in}_{(p, \ldots, p, p+q, p, \ldots, p)}\left(\widehat{I_{A}}\right)$ we are done. The ring $\mathbb{C}[x, \xi] / \operatorname{in}_{(p, \ldots, p, p+q, p, \ldots, p)}\left(\widehat{I_{A}}\right)$ is a graded ring. We must see that the element $\sigma_{L}(A \theta-\beta)$ is a not a zero divisor, that is, if $h \sigma_{L}(A \theta-\beta) \in$ $\operatorname{in}_{(p, \ldots, p, p+q, p, \ldots, p)}\left(\widehat{I_{A}}\right)$, then $h \in \operatorname{in}_{(p, \ldots, p, p+q, p, \ldots, p)}\left(\widehat{I_{A}}\right) . \operatorname{But}_{(p, \ldots, p, p+q, p, \ldots, p)}\left(\widehat{I_{A}}\right)$ is a monomial graded ideal, so for all the monomials in $h x_{1} \xi_{1} \in \operatorname{in}_{(p, \ldots, p, p+q, p, \ldots, p)}\left(\widehat{I_{A}}\right)$ and $h x_{n} \xi_{n} \in \operatorname{in}_{(p, \ldots, p, p+q, p, \ldots, p)}\left(\widehat{I_{A}}\right)$, then $h \in \operatorname{in}_{(p, \ldots, p, p+q, p, \ldots, p)}\left(\widehat{I_{A}}\right)$.

Then as we saw before,

$$
C h^{L}\left(\mathcal{H}_{A}(\beta)\right)=\mathcal{V}\left(\xi_{1} \xi_{n}, \xi_{2}, \ldots, \xi_{n-1}, x_{1} \xi_{1}, x_{n} \xi_{n}\right) .
$$

This is also true for any filtration $L$ defined with respect to $x_{n}=0$ if $L<a_{n-2} F+$ $\left(a_{n-1}-a_{n-2}\right) V$, so we have proved the parts (i) and (ii).

To see the (iii) part, we now fix a filtration $L$, defined with respect to the hyperplane $x_{n}=0$, and such that $L>a_{n-2} F+\left(a_{n-1}-a_{n-2}\right) V$. We take the elements in the toric ideal $I_{A}$ :

$$
C_{1, n-1}=\partial_{1}^{a_{n-2}} \partial_{n-1}^{b_{1}}-1, \quad C_{2, n}=\partial_{2}^{a_{n-1}}-\partial_{n}^{a_{1}}, \ldots, C_{n-1, n}=\partial_{n-1}^{a_{n-1}}-\partial_{n}^{a_{n-2}} .
$$

These elements form a $\mathbb{Q}$-basis of $\operatorname{ker}(A)$. We call $\widehat{I_{A}^{\prime}}$ the ideal generated by $C_{1, n-1}, C_{2, n}, \ldots, C_{n-1, n}$. The $L$-symbols of those elements form a regular sequence, they generate $\operatorname{in}_{(p, \ldots, p, p+q)}\left(\widehat{I_{A}^{\prime}}\right)$, and the $L$-symbol of the homogeneity operator is a regular sequence over $\mathbb{C}[x, \xi] / \operatorname{in}_{(p, \ldots, p, p+q)}\left(\widehat{I_{A}^{\prime}}\right)$. We have again that the fake initial ideal is equal to the initial ideal of the hypergeometric system. Hence

$$
C h^{L}\left(\mathcal{H}_{A}(\beta)\right)=\mathcal{V}\left(\xi_{1} \xi_{n-1}, \xi_{2}, \ldots, \xi_{n-2}, \xi_{n}, x_{1} \xi_{1}, x_{n-1} \xi_{n-1}\right) .
$$


The only case we have not studied yet is the one with more than one element negative and more than one element positive. To simplify notation we shall asume $A$ is a $1 \times(m+n)$ integer matrix with all its elements different and ordered.

Theorem 4.2. Given a $1 \times(m+n)$ integer matrix $A$ and $\beta \in \mathbb{C}$, such that $A=$ $\left(-b_{m} \ldots-b_{1} a_{1} a_{2} \ldots a_{n}\right)$, with $0<a_{1}<a_{2}<\ldots<a_{n}$, and $0<b_{1}<b_{2}<\ldots<$ $b_{m}$, the hypergeometric system $H_{A}(\beta)$ has no slopes with respect to the hyperplanes $x_{i}=0,1<i<m+n$, and it has only the slope $\frac{b_{m-1}}{b_{m-1}-b_{m}}$ with respect to the hyperplane $x_{1}=0$ and the slope $\frac{a_{n-1}}{a_{n-1}-a_{n}}$ with respect to $x_{m+n}=0$. The singular locus of the system is $\left\{x_{1}=x_{m+n}=0\right\}$.

(i) If we consider a filtration $L=p F+q V, p>0$, with $V$ defined with respect to $x_{i}=0,1<i<m+n$, then

$$
C h^{L}\left(\mathcal{H}_{A}(\beta)\right)=\mathcal{V}\left(\xi_{1} \xi_{m+n}, \xi_{2}, \ldots, \xi_{m+n-1}, x_{1} \xi_{1}, x_{m+n} \xi_{m+n}\right) .
$$

(ii) If we consider a filtration $L=p F+q V, p>0$, with $V$ defined with respect to $x_{1}=0$, with $L<b_{m-1} F+\left(b_{m}-b_{m-1}\right) V$, or a filtration with respect to $x_{m+n}=0$, with $L<a_{n-1} F+\left(a_{n}-a_{n-1}\right) V$, then

$$
C h^{L}\left(\mathcal{H}_{A}(\beta)\right)=\mathcal{V}\left(\xi_{1} \xi_{m+n}, \xi_{2}, \ldots, \xi_{m+n-1}, x_{1} \xi_{1}, x_{m+n} \xi_{m+n}\right) .
$$

(iii) If the filtration $L$ is defined with respect to the hyperplane $x_{1}=0$ and $L>b_{m-1} F+\left(b_{m}-b_{m-1}\right) V$, then

$$
C h^{L}\left(\mathcal{H}_{A}(\beta)\right)=\mathcal{V}\left(\xi_{2} \xi_{m+n}, \xi_{1}, \xi_{3}, \ldots, \xi_{m+n-1}, x_{2} \xi_{2}, x_{m+n} \xi_{m+n}\right) .
$$

(iv) If the filtration $L$ is defined with respect to the hyperplane $x_{m+n}=0$, such that $L>a_{n-1} F+\left(a_{n}-a_{n-1}\right) V$, then

$$
C h^{L}\left(\mathcal{H}_{A}(\beta)\right)=\mathcal{V}\left(\xi_{1} \xi_{m+n-1}, \xi_{2}, \ldots, \xi_{m+n-2}, \xi_{m+n}, x_{1} \xi_{1}, x_{m+n-1} \xi_{m+n-1}\right)
$$

Proof. First, we prove part (i). Let $L$ be a filtration defined with respect to the hyperplane $x_{i}=0$ for $1<i<m+n$. We consider the set of elements in the toric ideal:

$$
\begin{gathered}
C_{1, m+n}=\partial_{1}^{a_{n}} \partial_{m+n}^{b_{m}}-1, C_{1,2}=\partial_{1}^{b_{m-1}}-\partial_{2}^{b_{m}}, \ldots, C_{1, m}=\partial_{1}^{b_{1}}-\partial_{m}^{b_{m}}, \\
C_{m+1, m+n}=\partial_{m+1}^{a_{n}}-\partial_{m+n}^{a_{1}}, \ldots, C_{m+n-1, m+n}=\partial_{m+n-1}^{a_{n}}-\partial_{m+n}^{a_{n-1}} .
\end{gathered}
$$

They form a $\mathbb{Q}$-basis of $\operatorname{ker}(A)$, and we have an element of the form $\partial^{u}-1$, so we let $\widehat{I_{A}}$ be the ideal generated by $C_{1, m+n}, C_{1, i}$ with $1<i<m+1$, and $C_{j, m+n}$ with $m<j<m+n$. For any element in the toric ideal $\partial^{\mathbf{u}^{+}}-\partial^{\mathbf{u}^{-}}$, there always exist $\mathbf{v}=\mathbf{v}^{+}-\mathbf{v}^{-}$and $a \in \mathbb{N}$ such that $\partial^{\mathbf{v}^{+}}-\partial^{\mathbf{v}^{-}} \in \widehat{I_{A}}$, and $a \mathbf{u}=\mathbf{v}$. It is also clear that the $L$-symbols of those operator form a regular sequence, so they generate $\operatorname{gr}^{L}\left(\widehat{I_{A}}\right)$.

We can easily see that the $L$-symbol of the homogeneity operator is a regular sequence over $\mathbb{C}[x, \xi] / \operatorname{gr}^{L}\left(\widehat{I_{A}}\right)$, with a similar argument as the one used in the proof of lemma 4.1. This implies that the fake initial ideal is equal to the initial ideal, so we obtain that

$$
C h^{L}\left(\mathcal{H}_{A}(\beta)\right)=\mathcal{V}\left(\xi_{1} \xi_{m+n}, \xi_{2}, \ldots, \xi_{m+n-1}, x_{1} \xi_{1}, x_{m+n} \xi_{m+n}\right) .
$$

To prove the (ii) part, the same argument is valid for any filtration $L$ defined for $x_{1}=0$ if $L<b_{m-1} F+\left(b_{m}-b_{m-1}\right) V$, or defined for the hyperplane $x_{m+n}=0$ if $L<a_{n-1} F+\left(a_{n}-a_{n-1}\right) V$. 
We now consider a filtration $L$ defined for $x_{1}=0$ such that $L>b_{n-1} F+$ $\left(b_{n}-b_{n-1}\right) V$, to prove part (iii). We take the elements in the toric ideal:

$$
\begin{gathered}
C_{2, m+n}=\partial_{2}^{a_{n}} \partial_{m+n}^{b_{m-1}}-1, C_{1,2}=\partial_{1}^{b_{m-1}}-\partial_{2}^{b_{m}}, \ldots, C_{1, m}=\partial_{1}^{b_{1}}-\partial_{m}^{b_{m}}, \\
C_{m+1, m+n}=\partial_{m+1}^{a_{n}}-\partial_{m+n}^{a_{1}}, \ldots, C_{m+n-1, m+n}=\partial_{m+n-1}^{a_{n}}-\partial_{m+n}^{a_{n-1}} .
\end{gathered}
$$

Those elements form a $\mathbb{Q}$-basis for $\operatorname{ker}(A)$. Let $\widehat{I_{A}^{\prime}}$ be the ideal generated by those elements. Their $L$-symbols form a regular sequence, and we see as before that the $L$-symbol of the homogeneity operator is a regular sequence over $\mathbb{C}[x, \xi] / \operatorname{gr}^{L}\left(I_{A}\right)$. Then,

$$
C h^{L}\left(\mathcal{H}_{A}(\beta)\right)=\mathcal{V}\left(\xi_{2} \xi_{m+n}, \xi_{1}, \xi_{3}, \ldots, \xi_{m+n-1}, x_{2} \xi_{2}, x_{m+n} \xi_{m+n}\right) .
$$

With analogous arguments we obtain the same result for a filtration $L$ defined for $x_{m+n}=0$, and $L>a_{n-1} F+\left(a_{n}-a_{n-1}\right) V$. If we consider the ideal generated by $C_{1, m+n-1}, C_{1, i}$ with $1<i<m+1$ and $C_{j, m+n}$ with $m<j<m+n$, we get a very similar argument and obtain

$$
C h^{L}\left(\mathcal{H}_{A}(\beta)\right)=\mathcal{V}\left(\xi_{1} \xi_{m+n-1}, \xi_{2}, \ldots, \xi_{m+n-2}, \xi_{m+n}, x_{1} \xi_{1}, x_{m+n-1} \xi_{m+n-1}\right) .
$$

It is clear that for both cases in this section, if we have repeated elements, we can always calculate its slopes with these results and the arguments of the precedent section.

\section{REFERENCES}

[1] Adolphson, A. Hypergeometric functions and rings generated by monomials. Duke Mathematical Journal 73 (1994), 269-290. MR1262208 (96c:33020)

[2] Assi, A., Castro-Jiménez, F. J. and Granger, J. M. How to calculate the slopes of a D-module. Compositio Math. 104 (1996) 107-123. MF1421395 (98i:32010)

[3] Castro-Jiménez, F. J. and Takayama, N. Slopes of a Hypergeometric System Associated to a Monomial Curve. Trans. Amer. Math. Soc. 355 (2003), no. 9, 3761-3775. MR1990172 (2004j:32009)

[4] Gel'fand, I. M., Zelevinski, A. V. and Kapranov, M. M. Hypergeometric functions and toric varieties. Funktsional. Anal. i Prilozhen. (2) 23 (1989), 12-26; translation in Funct. Anal. Appl. (2) 23 (1989), 94-106. MR1011353 (90m:22025)

[5] Granger, J.M., Maisonobe, P. A basic course of differential modules. Élément de la theorie des systémes différentiels, $\mathcal{D}$-modules cohérents et holonomes. Travaux en Cours, 45, Hermann, Paris, (1993), 103-168. MF.1603676 (98h:32002)

[6] Hartillo-Hermoso, M. I. Slopes of hypergeometric systems of codimension one. Rev. Mat. Iberoamericana. 19 (2003), no. 2, 455-466 MR2023195

[7] Hotta, R. Equivariant D-modules. Proceedings of ICPAM Spring School in Wuhan, edited by P. Torasso. Travaux en Cours, Hermann, Paris, to appear, math.RT/9805021, (1991).

[8] Ince, E. L. Ordinary Differential Equations. Dover Publications, New York (1956). MR.0010757(6:65f)

[9] Laurent, Y. Polygone de Newton et b-fonctions pour les modules microdifférentiels. Ann. Sci. École Norm. Sup. (4), 20 (1987), 391-441. MR0925721 (89k:58282)

[10] Laurent, Y. and Mebkhout, Z. Pentes algébriques et pentes analytiques d'un $\mathcal{D}$-module. Ann. Sci. École Norm. Sup. (4) 32 (1999), 39-69. MR.1670595 (2001b:32015)

[11] Laurent, Y. and Mebkhout, Z. Image inverse d'un $\mathcal{D}$-module et polygone de Newton. Prépublication de l'Institut Fourier no 514 (2000).

[12] Mebkhout, Z. Le formalisme des six opérations de Grothendieck pour les $\mathcal{D}_{X}$-modules cohérent. Travaux en cours 35. Hermann, Paris (1989). MR1008245 (90m:32026)

[13] Mebkhout, Z. Le théorème de positivité de l'irregularité pour le $\mathcal{D}$-modules. Grothendieck Festschrift III, Progress in Math. 88 (1990), 84-131. MF.1106912(92j:32031) 
[14] Saito, M., Sturmfels, B. and Takayama, N. Gröbner deformations of hypergeometric differential equations. Algorithms and Computations in Mathematics 6, Springer (2000). MR1734566 (2001i:13036)

[15] Sturmfels, B. Gröbner Bases and Convex Polytopes. University Lecture Notes 8. American Mathematical Society, Providence (1996). MR1363949|(97b:13034)

Departamento de Matemáticas, Universidad de Cádiz, Aptdo. 40, Puerto Real 11510 (CÁdiz), SpAin

E-mail address: isabel.hartillo@uca.es 